\title{
Tempo de reação simples de jogadores de futebol de diferentes categorias e posições
}

\section{Simple reaction time in soccer players from differing categories and field positions}

\author{
C. Ruschel, A. Haupenthal, M. Hubert, H.B. Fontana, S.M. Pereira, H. Roesler
}

\begin{abstract}
Este estudo teve como objetivo analisar o tempo de reação simples (visual e auditivo) de jogares de futebol de diferentes categorias e posições. A amostra foi composta por 49 jogadores de futebol do sexo masculino, de diferentes categorias (amador e profissional) e posições (goleiro, zagueiro, lateral, volante, meio-campo e atacante). Para a coleta de dados utilizou-se um sistema composto por um interruptor, um dispositivo para emissão do estímulo visual ou sonoro e um software de aquisição. Para análise dos dados utilizou-se a estatística descritiva e inferencial $(p<.05)$. Na comparação entre as categorias, não houve diferença para o tempo de reação visual (TRV), enquanto os profissionais apresentaram tempos de reação auditiva (TRA) significativamente menores do que os amadores. Quanto às posições em campo, os goleiros apresentaram valores de TRV significativamente menores do que os meio-campistas, e não houve diferença para o TRA. Acredita-se que as principais diferenças entre jogadores de diferentes categorias e de diferentes posições estejam relacionadas a estratégias mais complexas de captação e utilização da informação visual em situações específicas da modalidade. Quanto ao TRA, a reação a estímulos auditivos pode não ser tão importante no desempenho no futebol, que é composto de estímulos predominantemente visuais.

Palavras-chave: tempo de reação simples, futebol, desempenho psicomotor
\end{abstract}

ABSTRACT

This study aimed at analyzing the visual and auditory simple reaction times in soccer players from differing categories and field positions. Participated in the study 49 male soccer players, amateurs and professionals, occupying the field positions of goalkeeper, center defender, right and left defender, halfback, midfielder, and forward. Data collection was carried out through a system composed by a switch, a mechanism of visual and auditory stimuli and appropriate acquisition software. Data were analyzed through descriptive and inferential statistics $(p<.05)$. When comparing categories, there was no difference in visual reaction time (VRT). Professionals showed faster auditory reaction times (ART) than amateurs did. Regarding field positions, goalkeepers showed significantly faster VRT than midfielders and there was no difference in terms of ART. Main differences between players from differing categories and field positions may be due to the adoption of more complex strategies in retaining and using visual information in specific situations. ART may be less important in soccer, since this is essentially a visual game.

Keywords: simple reaction time, soccer, psychomotor performance

Submetido: 08.09.2009 | Aceite: 11.12.2010

Caroline Ruschel, Alessandro Haupenthal, Marcel Hubert, Heiliane B. Fontana, Suzana M. Pereira e Helio Roesler. Centro de Ciências da Saúde e do Esporte da Universidade do Estado de Santa Catarina, Florianópolis, SC, Brasil.

Endereço para correspondência: Caroline Ruschel, CEFID/UDESC, Laboratório de Pesquisas em Biomecânica Aquática, Rua Pascoal Simone, 358, CEP 88080-350 - Florianópolis, SC - Brasil.

E-mail: carolruschel@yahoo.com.br 
A cada ano que passa é crescente o número de estudos com o objetivo de identificar os fatores determinantes do desempenho em esportes de elite. $\mathrm{O}$ aumento das atividades de pesquisa é particularmente evidente no futebol, uma vez que nesse esporte a importância das ciências do esporte para o desenvolvimento da modalidade tem sido amplamente aceita (Williams \& Hodges, 2005). Reilly e Gilbourne (2003), porém, ressaltam que a maioria dos trabalhos relativos ao futebol tem seu foco voltado a aspectos fisiológicos, enquanto aspectos relacionados a outras disciplinas tradicionais das ciências do esporte - como a psicologia e a aprendizagem motora - têm sido pouco explorados.

Nos últimos anos, atletas, técnicos e cientistas têm discutido a importância de habilidades perceptivo-cognitivas no desempenho esportivo, particularmente nos níveis de prática mais elevados (Vayens, Lenoir, Williams, Mazyn, \& Philippaerts, 2007). No futebol, as situações do jogo requerem do praticante uma permanente atenção sobre os corpos interativos (jogadores, bola, relação do campo), que depende de coordenadas em constante mudança e do processamento mental antecipativo de movimentos rápidos (Silva, 2000). As constantes alterações, segundo Weineck (1999), estão diretamente relacionadas às mudanças rápidas de direção, às manobras realizadas para enganar o adversário $\mathrm{e}$ às variações de movimentos durante a partida. Ainda segundo este autor, o sucesso do jogador frente a esses fatores está intimamente ligado à sua capacidade de reagir na menor unidade de tempo possível a situações não-previstas.

Nesse contexto, o tempo de reação simples (TRS) - intervalo de tempo decorrido desde um estímulo (que pode ser auditivo ou visual) até o início de uma resposta (Magill, 2000; Schmidt \& Wrisberg, 2001) - é há anos utilizado por pesquisadores como uma medida do desempenho sensório-motor (Lefthus, 1981).
O tempo de reação tem papel crucial em esportes como o futebol, no qual os jogadores enfrentam um ambiente complexo que é constantemente modificado e necessitam captar informações relativas à bola, aos companheiros de time e aos adversários (Williams, 2000). Atrasos na reação podem ser de importância crítica na determinação do sucesso em habilidades rápidas, tais como antecipar-se ao adversário em uma roubada de bola ou interceptar um chute a gol (Schmidt \& Wrisberg, 2001).

$\mathrm{Na}$ literatura, há poucos estudos que analisaram o tempo de reação de jogadores de futebol. Montés-Micó, Bueno, Candel e Pons (2000), e Ando, Kida e Oda (2001) compararam o TRS visual de jogadores de futebol e sujeitos não-atletas, encontrando valores menores para os futebolistas. Senel e Eroglu (2006) investigaram a correlação entre o tempo de reação e a velocidade de corrida em 20 metros. Outros estudos, investigaram, dentre outras variáveis, o tempo de reação de goleiros em situações de defesa simulada de penalidades (McMorris \& Colenso, 1996; McMorris, Copeman, Corcoran, Saunders, \& Potters, 1993).

Uma discussão remanescente em se tratando do TRS é a influência da experiência ou quantidade de prática do indivíduo na sua capacidade de reação. Em modalidades variadas, os estudos têm mostrado resultados divergentes. Alguns deles encontraram tempos de reação menores para atletas experientes quando comparados a atletas novatos (Bhanot \& Sidhu, 1979; Kioumourtzoglou, Kourtessis, Michalopoulou, \& Derri, 1998; Layton, 1991; Lowdon \& Pateman, 1980), enquanto outros relataram não haver diferença entre os níveis de prática (Helsen \& Starkes, 1999; Mori, Ohtani, \& Imanaka, 2002; Vaghetti, Roesler, \& Andrade, 2007).

Outro fator a ser considerado, além da experiência dos atletas, é o fato de os jogadores assumirem diferentes funções em campo. Além de haver características fisiológicas próprias de 
cada posição, as ações técnicas/táticas do jogador dependem invariavelmente da sua posição/função dentro de campo (Barros \& Guerra, 2004; Guerra, Soares, \& Burini, 2001). Bhanot e Sidhu (1980), ao analisarem o tempo de reação de jogadores de hóquei de acordo com a posição assumida em campo, afirmam que o TRS é uma variável que sofre influência da atividade específica realizada pelo indivíduo. Pouco se sabe a respeito de semelhanças ou diferenças entre os tempos de reação quando comparados jogadores de diferentes funções no futebol. Além disso, a grande maioria das informações veiculadas em livros e manuais para técnicos dizem respeito à importância do TRS para os goleiros, sem considerar as demais funções assumidas dentro de campo (Aoki, 2002; Domingues, 1997; Melo, 1997).

Diante das considerações supracitadas, este estudo teve como objetivo analisar o tempo de reação simples (visual e auditivo) de jogares de futebol de diferentes categorias e posições.

\section{MÉTODO}

\section{Amostra}

Participaram deste estudo descritivo 49 jogadores de futebol do sexo masculino, de diferentes categorias e posições, integrantes das equipes Juvenil e Júnior $(n=37$, com idade de $16.4 \pm .9$ anos e tempo de prática na modalidade de $5.4 \pm 1.9$ anos), representando a categoria de base, e da equipe Profissional "B" $(n=12$, com idade de $21.7 \pm 1.0$ anos e tempo de prática na modalidade de $11.8 \pm 3.4$ anos), representado os jogadores mais experientes, de um clube que disputava a Série "A" do Campeonato Brasileiro de Futebol. O estudo foi aprovado pelo Comitê de Ética em Pesquisa em Seres Humanos da Universidade do Estado de Santa Catarina e os participantes concordaram em participar da pesquisa mediante assinatura do Termo de Consentimento Livre e Esclarecido.

\section{Instrumentos}

Como instrumento foi utilizado o sistema proposto por Vaghetti et al. (2007), composto por um interruptor com uma tecla de sensibilidade de $.8 \mathrm{~N}$; um L.E.D. (light emitter diode) para emissão do estímulo visual; uma caixa acústica com frequência de $315 \mathrm{~Hz}$ e pressão sonora de até $81 \mathrm{~dB}$ para emissão do estímulo auditivo; e um dispositivo sincronizador de sinais visual, auditivo e digital. $\mathrm{O}$ sistema foi conectado a um microcomputador através de uma placa conversora $\mathrm{A} / \mathrm{D}$ CIODAS16/JR. A aquisição e o processamento dos dados foram realizados com a utilização do software SAD 32 (Silva \& Zaro, 1997).

\section{Procedimentos}

Os sujeitos foram orientados a se posicionar sentados em uma cadeira com o antebraço apoiado sobre uma mesa e com o dedo indicador sobre a tecla do interruptor (Figura 1) e receberam instruções para pressionar a tecla o mais rápido possível após perceberem os estímulos visual ou auditivo. Um pré-teste para cada situação foi realizado com o objetivo de familiarizar os indivíduos com os instrumentos de medida.

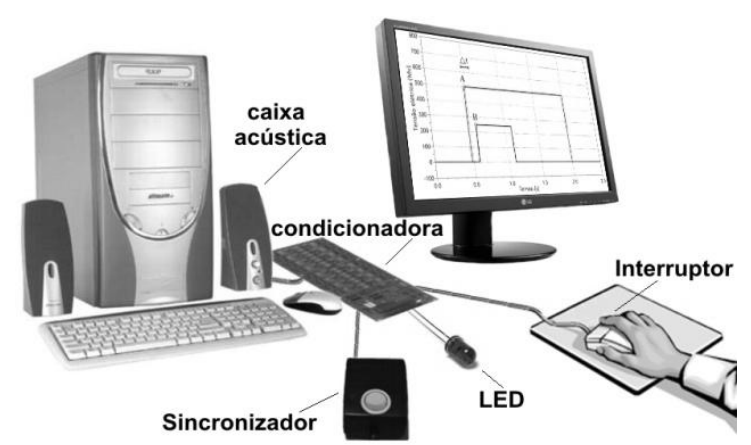

Figura 1. Representação esquemática do sistema de aquisição de dados

Após o período de familiarização, cada jogador realizou seis execuções do teste para investigação do tempo de reação simples manual visual (TRV) e seis execuções do teste para investigação do tempo de reação simples manual auditivo (TRA). A ordem das execuções foi pré-estabelecida por sorteio para que o treinamento da tarefa não influenciasse nos resultados. $O$ intervalo entre cada execução variou de 30 segundos a 1 minuto. 
Todos os sujeitos eram destros e realizaram os testes com a mão dominante.

$O$ equipamento sincronizador ficou sob controle do pesquisador e, quando acionado, gerou um sinal visual e um sinal de sincronismo (quando investigado o tempo de reação simples visual) ou um sinal auditivo e um sinal de sincronismo (quando investigado o tempo de reação simples auditivo). A representação gráfica da mensuração do tempo de reação simples está apresentada na Figura 2.

$O$ ponto "A" na curva do sincronizador (linha tracejada) representa o instante de acionamento do equipamento emissor de luz ou som (sinal visual ou sonoro) e a alteração de corrente captada pelo sistema; o ponto "B" na curva do interruptor (linha sólida) representa o instante no qual o sujeito pressionou a tecla após receber o estímulo. O intervalo representado por " $\Delta \mathrm{t}$ " equivale ao tempo de reação simples (em milissegundos) do sujeito.

Para a comparação dos resultados, os dados foram agrupados de acordo com dois critérios: categoria/nível de prática e posição dos jogadores em campo. Para o critério "categoria/nível de prática" foram caracterizados dois grupos: grupo dos amadores, composto por jogadores das categorias de base (juvenis e juniores), com idade inferior ou igual a 20 anos $(n=37)$; e grupo dos profissionais, composto por jogadores profissionais, com idade superior a 20 anos $(n=12)$. Para essa caracterização foram considerados fatores como tempo de dedicação à prática da modalidade (através da identificação da média dos anos de prática regular, que foi de $5.4 \pm 1.9$ anos para o grupo dos amadores e $11.8 \pm 3.4$ anos para o grupo dos profissio-nais) e volume de treinamento semanal (através da identificação da quantidade de sessões de treino por semana e da duração média de cada sessão, que correspondeu a 6 sessões de 2-3 horas cada para o grupo dos amadores e a 12 sessões de 23 horas cada para o grupo dos profissionais). Essas informações foram obtidas através de um formulário individual, que foi preenchido pelos jogadores antes do início dos testes. Para o critério "posição dos jogadores em campo", foram caracterizados seis grupos: goleiros/ guarda-redes $(n=12)$, zagueiros/defesa central $(n=5)$, laterais $(n=8)$, volantes/ médio defensivo $(n=11)$, meio-campistas/ médio ofensivo $(n=6)$ e atacantes/avançado $(n=7)$.

\section{Análise Estatística}

Utilizou-se para a apresentação dos dados a estatística descritiva, com a identificação dos valores de média e desvio padrão. A normalidade dos dados foi verificada através

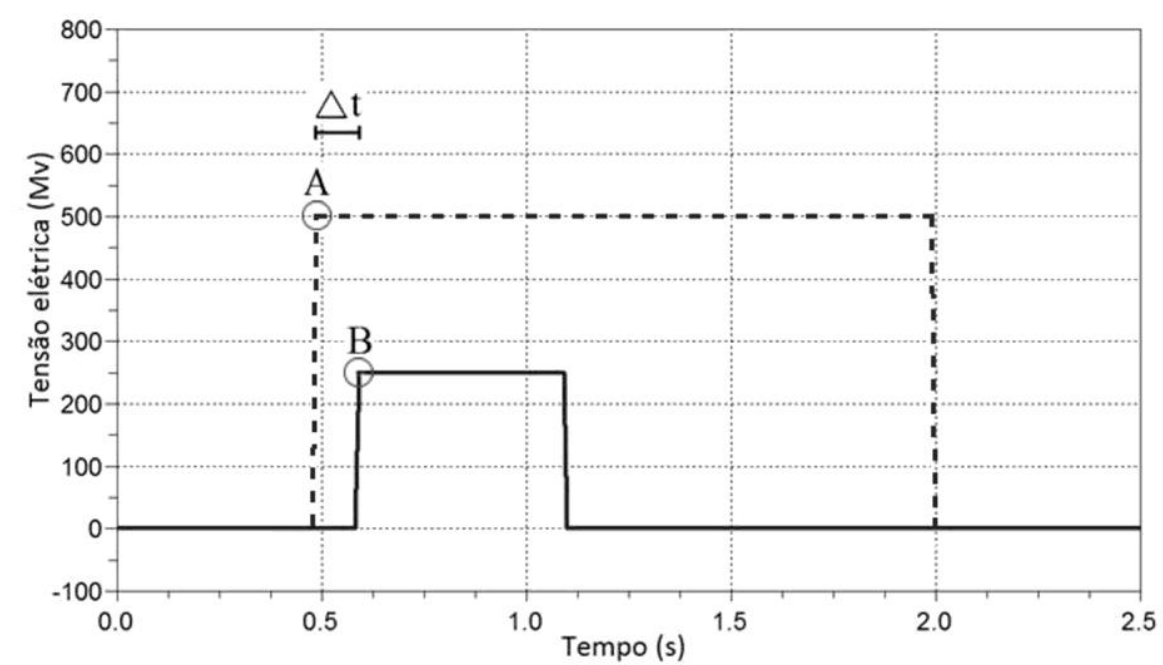

Figura 1. Representação gráfica da mensuração do tempo de reação simples de um sujeito da amostra. Nota: $\mathrm{A}=$ instante de acionamento do estímulo; $\mathrm{B}=$ instante de resposta do sujeito; $\Delta \mathrm{t}=$ tempo de reação simples 
do teste de Shapiro-Wilk, e a homogeneidade foi verificada através do teste de Levene, possibilitando a aplicação de testes paramétricos: (a) teste " $t$ " de Student para dados não pareados para a comparação dos tempos de reação simples entre os grupos caracterizados conforme o nível de prática dos sujeitos; (b) análise de variância (ANOVA one-way) e posthoc de Tukey para a comparação dos dados referentes aos grupos caracterizados pela posição dos jogadores em campo; e (c) teste "t" de Student para dados pareados para a comparação dos tempos de reação visual e auditivo para cada um dos grupos de sujeitos. O nível de confiança adotado para todos os testes foi de $95 \%(p<.05)$.

\section{RESULTADOS}

Na Tabela 1 são apresentados os valores de média e desvio padrão para o TRV e o TRA dos sujeitos do grupo dos amadores e do grupo dos profissionais, e o valor de $p$ obtido através da comparação das médias.

Os resultados do teste estatístico para a comparação das médias indicaram que os profissionais apresentaram tempos de reação auditivos significativamente menores do que os atletas da categoria de base $(p=.049)$. Em relação ao tempo de reação visual, apesar de menor para o grupo dos profissionais, não foi evidenciada diferença significativa entre os grupos $(p=.110)$. Para ambos os grupos os sujeitos apresentaram tempos de reação auditivos significativamente menores do que os tempos de reação visuais.

Na Tabela 2 constam os valores de média e desvio padrão para o TRV e o TRA dos sujeitos de acordo com a posição ocupada em campo, e o valor de $p$ obtido através da comparação das médias.

Tabela 1

Valores de média (e desvio padrão) para os tempos de reação simples visual (TRV) e auditivo (TRA) dos sujeitos amadores e profissionais e valores de $p$ obtidos nos testes estatísticos

\begin{tabular}{ccccc}
\hline Categoria & $n$ & TRV $(\mathrm{ms})$ & TRA $(\mathrm{ms})$ & $p^{(2)}$ \\
\hline Amadores & 37 & $204.9(26.6)$ & $183.7(33.9)$ & $.001^{*}$ \\
Profissionais & 12 & $190.3(27.9)$ & $162.0(28.5)$ & $.021^{*}$ \\
\hline$p^{(1)}$ & & .110 & $.049^{*}$ & - \\
\hline
\end{tabular}

Nota: $n$ = número de sujeitos; ${ }^{*}=$ diferença significativa no teste estatístico; $p^{(1)}$ : obtido na comparação entre os grupos de amadores e profissionais; $p^{(2)}$ : obtido na comparação do TRV com o TRA para cada um dos grupos

Tabela 2

Valores de média (e desvio padrão) para os tempos de reação simples visual (TRV) e auditivo (TRA) dos sujeitos de acordo com a posição ocupada em campo e valores de p obtidos nos testes estatísticos

\begin{tabular}{ccccc}
\hline Posição & $n$ & TRV $(\mathrm{ms})$ & TRA (ms) & $p^{(2)}$ \\
\hline Goleiro & 12 & $181.9(20.4)^{\mathrm{a}}$ & $157.4(32.1)$ & $.023^{*}$ \\
Zagueiro & 6 & $213.3(20.4)$ & $192.7(24.4)$ & .116 \\
Lateral & 7 & $203.6(22.6)$ & $176.4(25.6)$ & .063 \\
Volante & 11 & $200.9(33.4)$ & $183.3(39.1)$ & $.045^{*}$ \\
Meio-campo & 6 & $224.0(29.0)^{\mathrm{a}}$ & $205.5(37.0)$ & .600 \\
Atacante & 7 & $203.3(19.7)$ & $173.3(17.8)$ & $.018^{*}$ \\
\hline$p^{(1)}$ & & $.034^{*}$ & .065 & -
\end{tabular}

Nota: $n=$ número de sujeitos; ${ }^{*}=$ diferença significativa no teste estatístico; ${ }^{a}=$ grupos para os quais se verificou diferença significativa no post-hoc; $p^{(1)}$ : obtido na comparação entre os grupos de atletas conforme a posição; $p^{(2)}$ : obtido na comparação do TRV com o TRA para cada um dos grupos 
$\mathrm{Na}$ análise de variância para comparação das médias considerando-se as diferentes posições assumidas pelos jogadores foi verificada diferença significativa somente entre o grupo dos goleiros e dos meio-campistas para o TRV $(p=.020)$. Os goleiros, meio-campistas $\mathrm{e}$ atacantes apresentaram tempos de reação auditivos significativamente menores do que os tempos de reação visuais.

\section{DISCUSSÃO}

Conforme esperado, para a grande maioria dos grupos de jogadores, independentemente dos critérios de agrupamento (categoria ou posição), o tempo de reação ao estímulo visual foi significativamente maior do que o tempo de reação ao estímulo auditivo. Valores de TRA significativamente menores do que de TRV também são reportados por Bhanot e Sidhu (1979), Misra, Mahajan e Maini (1985), Senel e Eroglu (2006) e Vaghetti, Roesler e Andrade (2007).

Weineck (1999) afirma que o tempo de reação visual é mais longo que o tempo de reação auditivo, e as reações auditivas e visuais diferenciam-se entre si porque a mudança da energia da luz para impulsos neurais que podem ser levados ao cérebro pela retina é mais lenta que a transformação das ondas de energia em impulsos neuromusculares que alertam o sistema auditivo. De acordo com Bear, Connors e Paradiso (1996) e Guyton (1992), as vias do sistema visual são mais longas e o processo de captação da luz e de transformação em informação sensorial é mais complicado do que o processo realizado pelo sistema auditivo.

De acordo com Magill (2000), o tempo que um indivíduo leva para responder a um estímulo visual é de $250 \mathrm{~ms}$. Neste estudo, os valores do TRV foram consideravelmente menores do que aqueles sugeridos pelo autor, tanto para os jogadores amadores (204.9 ms), quanto para os jogadores profissionais (190.3 $\mathrm{ms})$. Entretanto, em outros estudos conduzidos com atletas de diferentes modalidades, os valores do TRV são menores do que aqueles propostos por Magill (2000) e semelhantes aos valores encontrados neste estudo. Soares, Osorno e Palafox (1987) encontraram médias de $192 \mathrm{~ms}$ para atletas de atletismo, $201 \mathrm{~ms}$ para jogadores de basquetebol, $200 \mathrm{~ms}$ para atletas de ginástica artística, $221 \mathrm{~ms}$ para nadadores e $225 \mathrm{~ms}$ para pugilistas, todos do sexo masculino.

Senel e Eroglu (2006) encontraram tempos de reação visual médios de $210 \mathrm{~ms}$, ao analisarem 104 jogadores de futebol profissional que atuavam na Liga de Futebol da Turquia. Montés-Micó et al. (2000), ao analisar o TRV de jogadores jovens de futebol, encontraram valores médios de $301 \mathrm{~ms}$ (sujeitos com 8 e 9 anos de idade), $256 \mathrm{~ms}$ (sujeitos 10 e 11 anos de idade) e de $207 \mathrm{~ms}$ (sujeitos com 12 e 13 anos idade). Neste estudo, observou-se a mesma tendência de valores maiores para os sujeitos de menor idade (amadores), em relação aos sujeitos mais velhos (profissionais).

Entretanto, não houve diferença significativa para o TRV entre os amadores e profissionais $(p=.110)$. Resultados semelhantes foram encontrados por Helsen e Starkes (1999), Mori et al. (2002), Soares et al. (1987) e Vaghetti et al. (2007), que compararam, respectivamente, os tempos de reação visual de jogadores de futebol, atletas de karaté, jogadores de basquetebol e surfistas com diferentes níveis de experiência na modalidade. Esses autores, apesar de não encontrarem diferenças significativas ao comparar os tempos de reação visual, observaram menores valores para os atletas mais experientes e com mais tempo de prática da modalidade. Outros autores, porém, encontraram valores de TRV significativamente menores para atletas mais experientes (Bhanot \& Sidhu, 1979; Kioumourtzoglou et al., 1998; Lowdon \& Pateman, 1980).

As médias para o tempo de reação auditivo são próximas ao valor proposto por Magill (2000) para a resposta de um indivíduo a um estímulo auditivo, que é $170 \mathrm{~ms}$, e também semelhantes à média encontrada por Senel e 
Eroglu (2006) para jogadores de futebol profissional, de $176 \mathrm{~ms}$. Quando comparados os atletas com diferentes níveis de prática, os resultados sugerem que os profissionais apresentam valores de TRA menores do que os amadores. Resultados semelhantes foram encontrados nos estudos de Bhanot e Sidhu (1979), comparando jogadores de hóquei experientes a novatos, e de Layton (1991), comparando atletas de karaté mais graduados a atletas menos graduados.

É importante considerar, porém, que a diferença entre os grupos no que se refere ao TRA se encontra no limiar da significância estatística $(p=.049)$. Além disso, o TRA pode não ser considerado uma variável determinante de desempenho no futebol, visto que nesta modalidade os estímulos visuais são prevalecentes, seja na análise das trajetórias da bola, seja na percepção das movimentações dos companheiros e/ou adversários (Garganta, 2001).

Ainda em se tratando da relação entre o tempo de reação simples e nível de prática, Chumura, Nazr e Kaciuba-Uscilko (1994) relataram em seu estudo que atletas de elite, quando submetidos a testes de esforço aeróbio, têm a habilidade de manter as condições psicomotoras estáveis, não aumentando os valores do TRS. Desta forma, no futebol, tão importante quanto perceber padrões complexos com rapidez e eficiência e utilizar estratégias refinadas para a antecipação das ações dos oponentes é desenvolver a capacidade de manter tais habilidades durante o maior tempo possível em uma partida, através dos programas de treinamento.

Outro fator importante a ser considerado é a discussão recente das diferenças entre atletas experientes e novatos no que diz respeito a variáveis perceptivo-cognitivas mais complexas do que o tempo de reação simples. Segundo Rezende e Valdés (2003), dentre as habilidades que caracterizam um atleta expert e que indicam que um atleta amador possui potencial para a profissionalização destacam-se: a percepção de padrões complexos com rapidez e eficiência, e a utilização de estratégias visuais refinadas que ampliam a capacidade de antecipação das ações dos oponentes.

Jogadores mais experientes não necessariamente reagem mais rápido do que jogadores novatos. Estudos recentes têm mostrado que atletas mais experientes são capazes (a) de perceber mais rapidamente a presença da bola em situações de jogo (Starkes, 1987), (b) de buscar de forma mais eficiente informações relevantes sobre partes do corpo do oponente e sobre o campo de jogo (Ripoll, Kerlirzin, Stein, \& Reine, 1995), (c) de antecipar a bola e a ação de um adversário com mais facilidade (Williams \& Davids, 1998), (d) de lembrar e reconhecer cenas de jogos/jogadas (Williams \& Davids, 1995), além de (e) ter um conhecimento maior das probabilidades de ocorrer determinada ação (Williams, 2000). Goleiros mais experientes, por exemplo, não têm tempos de reação menores do que goleiros novatos, mas apresentam maior acurácia na predição da altura e da direção da bola em uma cobrança de pênalti, pois utilizam informações relevantes como a posição do pé de apoio do batedor, ao invés de rápidas reações (Savelsbergh, Van der Kamp, Williams, \& Ward, 2005).

Quando considerada a posição dos jogadores em campo, o TRV dos goleiros foi significativamente menor dos que os valores encontrados para os meio-campistas. Embora os valores encontrados para os goleiros tenham sido menores do que os valores dos zagueiros, laterais, volantes e atacantes, a diferença não foi significativa.

Bhanot e Sidhu (1980), em um estudo comparativo entre os tempos de reação simples visual e auditivo de jogadores de hóquei de diferentes funções/posições, encontraram resultados semelhantes, sendo os maiores tempos de reação simples, tanto visual quanto auditivo, obtidos pelo grupo de jogadores de meio-campo.

Para o TRA não foram encontradas diferenças significativas quando comparadas as diferentes posições assumidas pelos jogadores 
em campo, mais uma vez sugerindo que esta não é uma variável determinante do desempenho no futebol, independentemente da posição assumida em campo.

Menores valores de TRV para os goleiros podem ser justificados pelo fato de que os programas específicos de treinamento para essa posição contemplam atividades de desenvolvimento/aperfeiçoamento do tempo de reação, diferentemente do programa de treinamento generalizado ao qual são submetidos os demais jogadores, sem diferenciação conforme seu posicionamento em campo.

Entretanto, é importante considerar que os procedimentos metodológicos utilizados nesse estudo são laboratoriais e não são específicos às tarefas desempenhadas pelos jogadores em campo. Acredita-se que, numa análise de habilidades perceptivo-cognitivas mais complexas, adaptada às condições de prática da modalidade, sejam observadas diferenças entre jogadores de diferentes posições, o que possibilitaria um melhor entendimento de fatores como tomada de decisão e adequação da resposta ao estímulo, determinantes das ações técnico/táticas a serem realizadas durante uma partida.

Em conclusão, nesse estudo foram analisados os tempos de reação simples visual e auditivo de jogadores de futebol de diferentes categorias e posições. Em se tratando das categorias analisadas (jogadores amadores $x$ profissionais), os resultados agregam evidências à literatura no que diz respeito à não existência de diferenças entre jogadores mais experientes e jogadores menos experientes, para o tempo de reação simples visual. Isso condiz com as afirmações recentes feitas por estudiosos da área, que acreditam que as principais diferenças entre experts e jogadores intermediários e/ou novatos estejam relacionadas a estratégias mais complexas de captação e utilização da informação visual em diferentes situações de jogo. Quanto à comparação dos jogadores de diferentes posições, os resultados demonstraram reações visuais mais rápidas dos goleiros somente em relação aos meio- campistas. É possível que uma variável como o tempo de reação simples não possa ser utilizada como parâmetro de comparação entre jogadores que desempenham funções diferentes em campo, uma vez que a diferença entre eles pode residir em variáveis mais complexas e específicas à tarefa desempenhada. Por fim, o tempo de reação auditivo foi diferente quando comparadas as categorias e semelhante quando comparadas as posições. Entretanto, ressalta-se que ao contrário do que acontece em modalidades como a natação e o atletismo, a reação a estímulos auditivos pode não ser tão importante no desempenho no futebol, que é composto de estímulos predominantemente visuais.

\section{REFERÊNCIAS}

Ando, S., Kida, N., \& Oda, S. (2001). Central and peripheral visual reaction time of soccer players and nonathletes. Perceptual and Motor Skills, $92(3), 786-794$.

Aoki, M. S. (2002). Fisiologia, treinamento e nutrição aplicada ao futebol. Jundiaí: Fontoura.

Barros, T. L., \& Guerra, I. (2004). Ciência do futebol. Barueri: Manole.

Bear, M. F., Connors, B. W., \& Paradiso, M. A. (1996). Neuroscience: Exploring the brain. Baltimore: Williams \& Wilkins.

Bhanot, J. L., \& Sidhu, L. S. (1979). Reaction time of Indian hockey players with reference to three levels of participation. Journal of Sports Medicine, 19, 199-204.

Bhanot, J. L., \& Sidhu L. S. (1980). Reaction time of hockey players with reference to their field positions. Journal of Sports Medicine, 20, 423-30.

Chumura, K., Nazr, K., \& Kaciuba-Uscilko, H. (1994). Choice reaction time during graded exercise in relation to blood lactate and plasma catecholamine thresholds. International Journal of Sports Medicine, 15, 172-176.

Domingues, A. (1997). Goleiro: 100 segredos. Curitiba: Verbo.

Garganta, J. (2001). O desenvolvimento da velocidade nos jogos desportivos coletivos. Lecturas Educación Física, 6(30), 1-11.

Guerra, I., Soares, E. A., \& Burini, R. C. (2001). Aspectos nutricionais do futebol de competição. Revista Brasileira Medicina do Esporte, 7(6), 200-206. 
Guyton, A. C. (1992). Tratado de fisiologia médica. Rio de Janeiro: Guanabara Koogan.

Helsen, W. F., \& Starkes, J. L. (1999). A multidimensional approach to skilled perception and performance in sport. Applied Cognitive Psychology, 13, 1-27.

Kioumourtzoglou, E., Kourtessis, T., Michalopoulou, M., \& Derri, V. (1998). Differences in several perceptual abilities between experts and novices in basketball, volleyball and waterpolo. Perceptual and Motor Skills, 86, 899-912.

Layton, C. (1991). How fast are the punches and kicks of traditional Shotokan karateka? Traditional Karate, 4, 29-31.

Lefthus, G. K. (1981). Sensorimotor performance and limb preference. Perceptual and Motor Skills, 52, 688-693.

Lowdon, B. J., \& Pateman, N. A. (1980). Physiological parameters of international surfers. Australian Journal of Sports Medicine, 12, 34-39.

Magill, R. A. (2000) Aprendizagem motora: Conceitos e aplicações. São Paulo: Edgard Blücher.

McMorris, T., \& Colenso, S. (1996). Anticipation of professional soccer goalkeepers when facing right- and left-footed penalty kicks. Perceptual and Motor Skills, 82, 931-934.

McMorris, T., Copeman, R., Corcoran, D., Saunders, G., \& Potters, S. (1993). Anticipation of soccer goalkeepers facing penalty kicks. In T. Reilly, J. Clarys, \& A. Stibbe (Eds.), Science and football II (pp. 250-253). London: E \& FN Spon.

Melo, R. S. (1997). Qualidades físicas e psicológicas e exercícios técnicos do atleta de futebol. Rio de Janeiro: Sprint.

Misra, N., Mahajan, K. K., \& Maini, B. K. (1985). Comparative study of visual and auditory reaction time of hands and feet in males and females. Indian Journal of Physiology and Pharmacology, 29(4), 213-218.

Montés-Micó, R., Bueno, I., Candel, J, \& Pons, A. M. (2000). Eye-hand and eye-foot visual reaction times of young soccer players. Optometry, 71 (12), 775-780.

Mori, S., Ohtani, Y., \& Imanaka, K. (2002). Reaction times and anticipatory skills of karate athletes. Human Movement Sciences, 21, 213-230.

Reilly, T., \& Gilbourne, D. (2003). Science and football: A review of applied research in the football codes. Journal of Sports Sciences, 21, 693705.

Rezende, A., \& Valdés, H. (2003). Métodos de estudo das habilidades táticas: Abordagem comparativa entre jogadores habilidosos e iniciantes. Lecturas Educación Física, 9(65), 1-11.

Ripoll, H., Kerlirzin, Y., Stein, J. F., \& Reine, B. (1995). Analysis of information processing, decision making, and visual strategies in complex problem solving sport situations. Human Movement Science, 14, 325-349.

Savelsbergh, G. J. P., Van der Kamp, J., Williams, A. M., \& Ward, P. (2005). Anticipation and visual search behavior in expert soccer goalkeepers. Ergonomics, 14, 1686-1697.

Schmidt, R. A., \& Wrisberg, C. A. (2001). Aprendizagem e performance motora. Porto Alegre: Artmed.

Senel, O., \& Eroglu, H. (2006). Correlation between reaction time and speed in elite soccer players. Journal of Exercise Science and Fitness, 4(2), 126130.

Silva, L. M., \& Zaro, M. A. (1997). Sistema de aquisição de dados SAD 2 Versão (3.0): Manual de operação. Porto Alegre: UFRGS-DEMEC.

Silva, V. F. (2000). Os efeitos de um programa de "treinamento de motricidade" na performance de atletas de futebol de diferentes níveis técnicos. Consultado em 11 de Novembro de 2004, a partir de http://www.personaltraining.com.br/ efeitosvernon.html

Soares, J., Osorno, L. A. N., \& Palafox, G. H. M. (1987). Estudo comparativo do tempo de reação visuo-manual simples em praticantes de esportes. Kinesis, 3, 77-85.

Starkes, J. (1987). Skill in field hockey: The nature of the cognitive advantage. Journal of Sport Psychology, 9, 146-160.

Vaeyens, R., Lenoir, M., Williams, A. M., Mazyn, L., \& Philippaerts, R. M. (2007). The effects of task constraints on visual search behavior and decision-making skill in youth soccer players. Journal of Sport \& Exercise Psychology, 29, 147169.

Vaghetti, C., Roesler, H., \& Andrade, A. (2007). Tempo de reação simples auditivo e visual em surfistas com diferentes níveis de habilidade: Comparação entre atletas profissio-nais, amadores e praticantes. Revista Brasileira de Medicina do Esporte, 13(2), 81-85.

Weineck, J. (1999). Treinamento ideal. São Paulo: Manole.

Williams, A. M. (2000). Perceptual skill in soccer: Implications for talent identification and development. Journal of Sports Sciences, 18, 737750 . 
$82 \mid$ C. Ruschel, A. Haupenthal, M. Hubert, H.B. Fontana, S.M. Pereira, H. Roesler

Williams, A. M., \& Davids, K. (1995). Declarative knowledge in sport: A byproduct of experience or a characteristic of expertise? Journal of Sport and Exercise Psychology, 17, 259-275.

Williams, A. M., \& Davids, K. (1998). Visual search strategy, selective attention, and expertise in soccer. Research Quarterly for Exercise and Sport, 69, 111-128.

Williams, A. M., \& Hodges, N. J. (2005). Practice, instruction and skill acquisition in soccer: Challenging tradition. Journal of Sports Sciences, 23(6), 637-650.

(cc) EY-No Todo o conteúdo da revista Motricidade está licenciado sob a Creative Commons, excepto quando especificado em contrário e nos conteúdos retirados de outras fontes bibliográficas. 